\title{
Les installations géothermiques de Bouillante
}

\section{The geothermal facilities of Bouillante}

\author{
Ph. Jaud \\ Direction \\ des Études et Recherches
}

\author{
D. Lamethe, \\ Direction régionale \\ des Départements d'Outre-Mer
}

Electricité de France

\begin{abstract}
Les travaux d'exploration réalisés en Guadeloupe jusqu'en 1977 par la Société EURAFREP ont permis de mettre en évidence l'existence d'un réservoir géothermique profond du type "eau chaude" à $240{ }^{\circ} \mathrm{C}$. Quatre forages ont été réalisés, dont deux se sont révélés producteurs de vapeur et, après plusieurs campagnes d'essais de productivité, Electricité de France a entrepris la réalisation productivité, Electricité de France a entrepris la réal
d'une centrale géothermique prototype de $4200 \mathrm{~kW}$.

Cette centrale utilise la technique du double flashing (deux étages de production de vapeur) et une turbine à condensation équipée d'un condenseur barométrique à mélange refroidi par l'eau de mer.

Cette centrale, fortement automatisée, fournira $6 \%$ de l'énergie électrique de l'ile à un coût proche de celui des Diesels existants.
\end{abstract}

The exploratory works completed in Guadeloupe in 1977 by EURAFREP demonstrated the existence of a deep geothermal reservoir of the "hot water" type at $40{ }^{\circ} \mathrm{C}$. Four drillings were made, two of which produced steam. After a few productivity test campaigns, Electricité de France undertook construction of a prototype $4200 \mathrm{~kW}$ geothermal power station.

This power station uses the double flashing technique (two steam production stages) and a condensation turbine equipped with a barometric mixing condenser cooled by sea water.

This highly automated power station will supply six percent of the electric power for Guadeloupe at a cost near that of the existing diesel generators.

\section{Introduction}

\begin{abstract}
A la vue des réalisations géothermiques de nos collègues italiens de l'E.N.E.L., au début des années soixante, E.D.F. a demandé une étude géologique destinée à savoir s'il existait en France métropolitaine une probabilité de découvrir un champ géothermique du type de celui qui existe à Larderello en Toscane.

Les résultats de cette étude et ceux des études ultérieures menées par le Bureau de recherches géologiques et minières (B.R.G.M.) ne semblent pas ouvrir de perspectives favorables pour le développement de la géothermie haute-énergie en France; par contre les nombreuses nappes d'eau chaude que renferme notre sous-sol national, en particulier sous les grands centres urbains de l'Aquitaine et de la Région Parisienne, ont permis de voir la réalisation d'une cinquantaine d'opérations de chauffage géothermique qui ont donné à la France une compétence technique importante dans ce domaine.
\end{abstract}

En ce qui concerne les départements français d'Outre-Mer, la situation de la géothermie haute-énergie est plus favorable puisque la découverte en Guadeloupe d'un réservoir exploitable d'eau chaude sous pression $\left(240^{\circ} \mathrm{C}\right)$ a permis la construction de la première centrale géothermique française de production d'électricité d'une puissance d'environ $5 \mathrm{MW}$ électriques.

Par ailleurs, il existe à la Martinique et dans l'île de la Réunion des indices géologiques qui laissent supposer l'existence de réservoirs géothermiques à haute température.

Cette première centrale française étant sur le point d'être mise en service, il est apparu intéressant, à l'occasion de ce congrès, de présenter les choix techniques retenus pour sa conception et d'estimer l'intérêt économique que la géothermie haute énergie peut apporter dans les départements d'Outre-Mer. 


\section{Historique de la recherche et du développement de la géothermie en Guadeloupe}

La possibilité de mettre en évidence une production de vapeur utilisable pour la génération d'électricité intéressa les Pouvoirs publics et, en 1963, à la demande de la Société de production d'électricité de Guadeloupe (S.P.D.E.G.), une étude préliminaire fut entreprise dans la région de Bouillante par le Bureau de recherches géologiques et minières.

Les données positives recueillies au cours de ces travaux incitèrent, en 1967, la Société Eurafrep à s'associer à la Société de production d'électricité de Guadeloupe pour entreprendre des recherches de vapeur géothermale.

Les travaux d'exploration profonde réalisés jusqu'en 1977 permirent alors de mettre en évidence l'existence d'un réservoir géothermique profond du type "eau chaude " à $240^{\circ} \mathrm{C}$.

Quatre forages ont été réalisés; deux se sont révélés être producteurs de vapeur :

- BO 2 qui recoupe une faille fortement productrice à la profondeur de 338 mètres, produit plus de $30 \mathrm{t} / \mathrm{h}$ de vapeur accompagnées de $120 \mathrm{t} / \mathrm{h}$ d'eau pour une pression de 6 bars;

- BO 4 qui atteint la profondeur de 2500 mètres, rencontre le réservoir sur plus de 2000 mètres, mais ne produit que $13 \mathrm{t} / \mathrm{h}$ de vapeur et $50 \mathrm{t} / \mathrm{h}$ d'eau pour une pression de 5 bars.

A partir des résultats obtenus, Électricité de France, qui a pris en 1975 la suite de la S.P.D.E.G., a décidé en 1978 d'engager l'étude d'une centrale géothermique d'une puissance nette de $4200 \mathrm{~kW}$.

Pour cette centrale prototype d'Électricité de France, la Direction Régionale chargée des Départements d'Outre-mer, maitre d'ouvrage, a confié la maîtrise d'œuvre à la Région d'Equipement Alpes-Marseille, les études étant réalisées par la Direction des Études et Recherches.

La construction du groupe turbo-alternateur a commencé en usine en 1981 et l'installation est maintenant dans sa phase définitive, le premier couplage étant prévu courant 1985.

\section{La centrale}

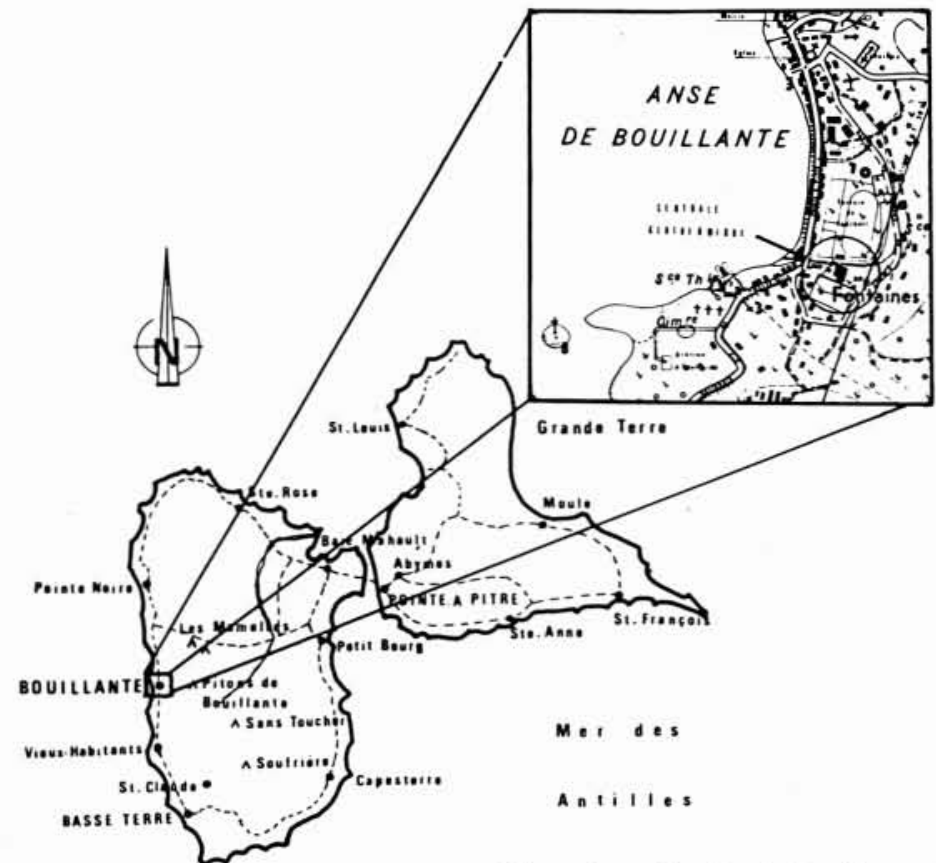

Figure 1. - Le site de Bouillante.

\section{Localisation du site}

Située à $7000 \mathrm{~km}$ de la métropole, dans l'Atlantique tropical, la Guadeloupe appartient à l'arc des petites Antilles.

Le terrain où est construit la centrale est situé sur la côte Ouest de l'île de Basse-Terre, au lieu dit : "Cocagne ", à environ 500 mètres au Sud du Centre de la commune de Bouillante et à une distance d'environ $15 \mathrm{~km}$ à vol d'oiseau du volcan de la Soufrière (voir carte ci-après).

La commune de Bouillante est soumise au régime des vents alizés soufflant de secteur Est, et sa position sur le versant sous-le-vent réduit les précipitations à moins de $1250 \mathrm{~mm}$ d'eau par an.

Le climat y est tropical et humide (température moyenne de l'ordre de $25^{\circ} \mathrm{C}$ tout au long de l'année) avec des passages cycloniques qui se produisent souvent entre juillet et octobre; c'est une zone à forte séismicité.

\section{Caractéristiques du réservoir géothermique}

L'exploration profonde de l'anomalie géothermique de Bouillante réalisée par la Société Eurafrep a montré que le sous-sol renferme une accumulation de chaleur considérable puisque des roches comprises entre 230 et $250^{\circ} \mathrm{C}$ ont été rencontrées entre 600 et 2500 mètres de profondeur, et que cette réserve de calories peut se développer plus bas 


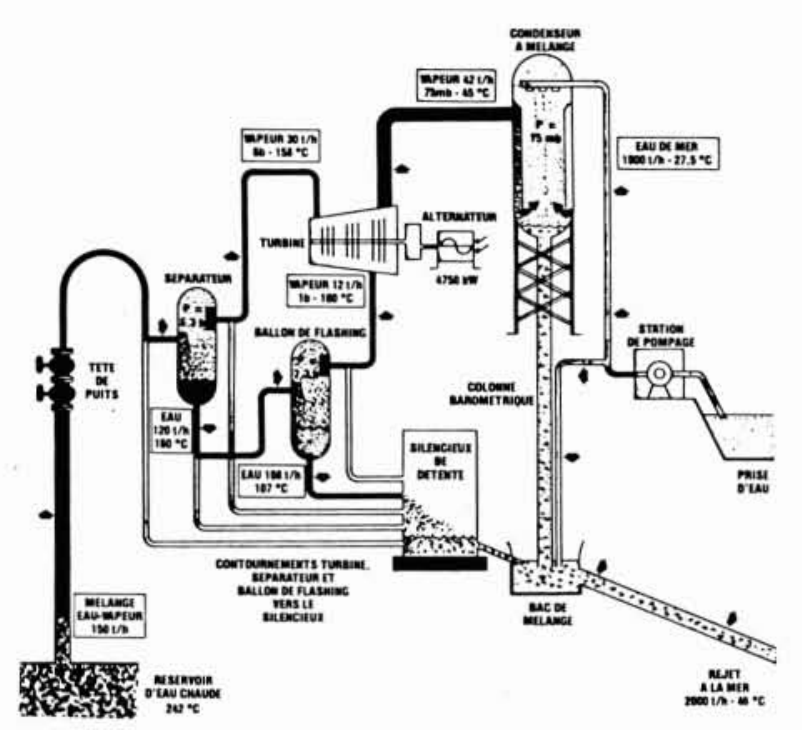

Figure 2. - Schéma de principe de la centrale.

encore. D'autre part, le réservoir profond devrait se prolonger vers le Sud au moins jusqu'à Thomas, c'est-à-dire sur une longueur minimum de $2,5 \mathrm{~km}$. Sa largeur reconnue étant de l'ordre de $1 \mathrm{~km}$, la surface du gisement serait d'au moins $2,5 \mathrm{~km}^{2}$.

Le réservoir géothermique de Bouillante est constitué par des sables et des tufs peu poreux, mais coupés de failles inclinées de direction Nord-Sud.

Les essais conduits sur les différents forages ont montré que seuls les forages $\mathrm{BO} 2$ et $\mathrm{BO} 4$ ont une production régulière d'un mélange eau-vapeur $(20 \%$ de vapeur pour $80 \%$ d'eau), le premier étant environ 3 fois plus producteur que le second et capable d'un débit total supérieur à $160 \mathrm{t} / \mathrm{h}$.

La composition chimique de l'eau géothermale en sortie du séparateur est très voisine pour les deux forages producteurs et se rapproche beaucoup de celle de Cerro-Prieto au Mexique, si ce n'est par la quantité beaucoup plus faible d'hydrogène sulfuré présent dans la vapeur.

Tableau I. - Composition chimique de l'eau géothermale en $\mathrm{mg} / \mathrm{l}$

\begin{tabular}{|c|c|c|r|r|r|c|}
\hline & $\mathrm{Cl}^{-}$ & $\mathrm{Na}^{+}$ & $\mathrm{K}^{+}$ & $\mathrm{Ca}^{2+}$ & $\mathrm{Si} \mathrm{O}_{2}$ & $\begin{array}{l}\text { Incondensables } \\
\text { dans la vapeur }\end{array}$ \\
\hline BO 2 & 16330 & 6700 & 1080 & 2750 & 670 & $0,4 \%$ \\
\hline BO 4 & 15590 & 6680 & 937 & 2275 & 670 & $0,04 \%$ \\
\hline Mexique & 14061 & 7641 & 1822 & 439 & 1093 & 1 à 3\% \\
\hline
\end{tabular}

\section{Caractéristiques techniques}

Il a été retenu d'exploiter au mieux l'énergie disponible en sortie du forage BO 2 et de garder BO 4 en secours en cas de déclin de la productivité du puits.

Après une étude détaillée des différentes possibilités de production d'électricité à partir de l'énergie géothermique produite par le forage $\mathrm{BO} 2$, les cycles à fluides volatils ont été abandonnés à cause des problèmes de dépôts de silice sur les surfaces d'échange et il a été retenu une installation constituée par un groupe turbo-alternateur à condensation alimenté par deux flux de vapeur saturée à des pressions de 6 bars et de 1 bar.

Le principe de fonctionnement de la centrale est le suivant (voir figure 2):

Le mélange eau-vapeur issu du forage est admis dans un séparateur-sécheur d'où sort d'une part la vapeur haute pression qui alimente la turbine à une pression de 6 bars, tandis que d'autre part l'eau géothermale séparée, à une température voisine de $160^{\circ} \mathrm{C}$, est envoyée dans un ballon de flashing pour produire la vapeur basse pression.

L'énergie potentielle de la vapeur se transforme en énergie mécanique dans la turbine, puis en énergie électrique dans l'alternateur. La vapeur d'échappement est ensuite condensée par l'eau de mer de refroidissement dans un condenseur à mélange avec mise en dépression barométrique.

L'eau géothermale résiduaire, à $100^{\circ} \mathrm{C}$, est diluée avec l'eau provenant du condenseur puis envoyée à la mer par un canal d'évacuation. Cette dilution permet de limiter la température des rejets à moins de $45^{\circ} \mathrm{C}$ et de réduire les dépôts.

La turbine fonctionne le plus souvent alimentée par les deux flux de vapeur haute et basse pression. Cependant elle peut tourner avec la vapeur haute pression seule, ce qui permet d'intervenir sur le ballon de flashing sans arrêter totalement la production d'électricité. L'eau géothermale provenant du premier ballon séparateur est alors envoyée vers un silencieux où elle se détend.

D'un point de vue géologique, il est important de garder un fonctionnement régulier du forage éventuellement à débit légèrement réduit. En cas d'indisponibilité de la centrale, arrêt volontaire ou déclenchement, des circuits de contournement d'eau et de vapeur permettent d'évacuer le débit du forage vers le silencieux. La vapeur s'échappe alors du silencieux à l'atmosphère et l'eau résiduaire est mélangée à l'eau du circuit de refroidissement avant son évacuation vers la mer.

Compte tenu de sa faible puissance et pour réduire les coûts d'exploitation, la centrale géothermique de Bouillante sera entièrement automatique. Elle fonctionnera en base sur le réseau de distribution de la Guadeloupe et fournira en permanence toute l'énergie disponible au forage. Si l'on admet qu'elle pourra fonctionner pendant 7000 heures par an, elle délivrera alors au réseau une énergie annuelle d'environ 29 millions de $\mathrm{kWh}$, qui représenteront pour la Guadeloupe $6 \%$ de l'énergie électrique produite en 1984 et une économie d'énergie d'environ 6600 TEP.

\section{Conception de la centrale}

La centrale est formée de deux ensembles distincts : 


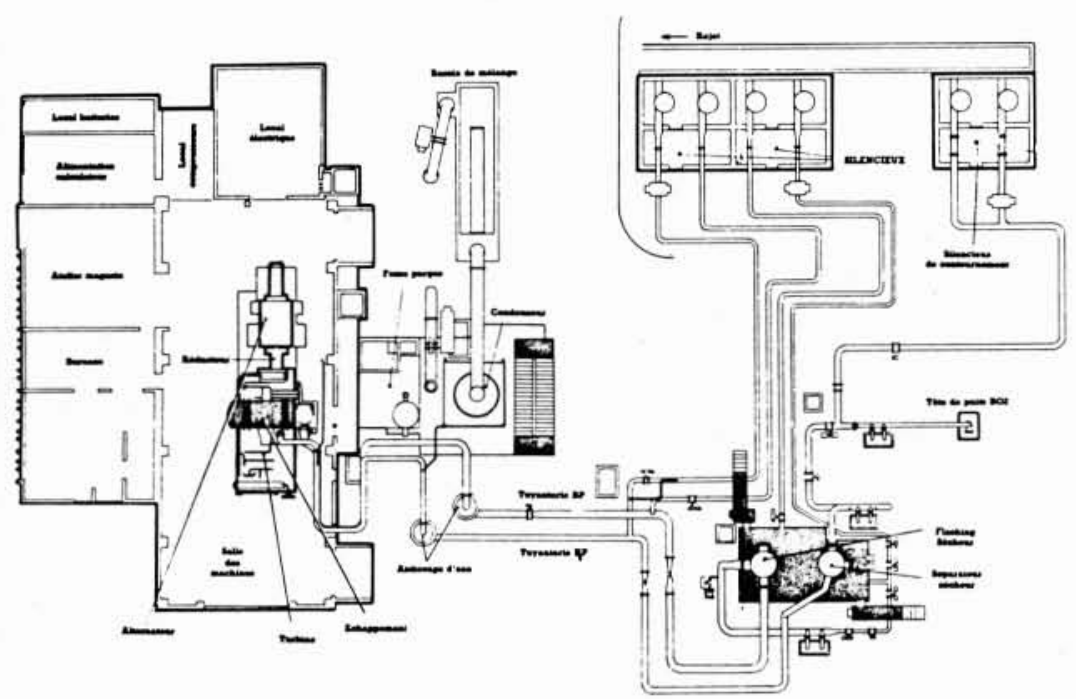

Figure 3. - Plan d'implantation du bâtiment usine et du système de production de vapeur.

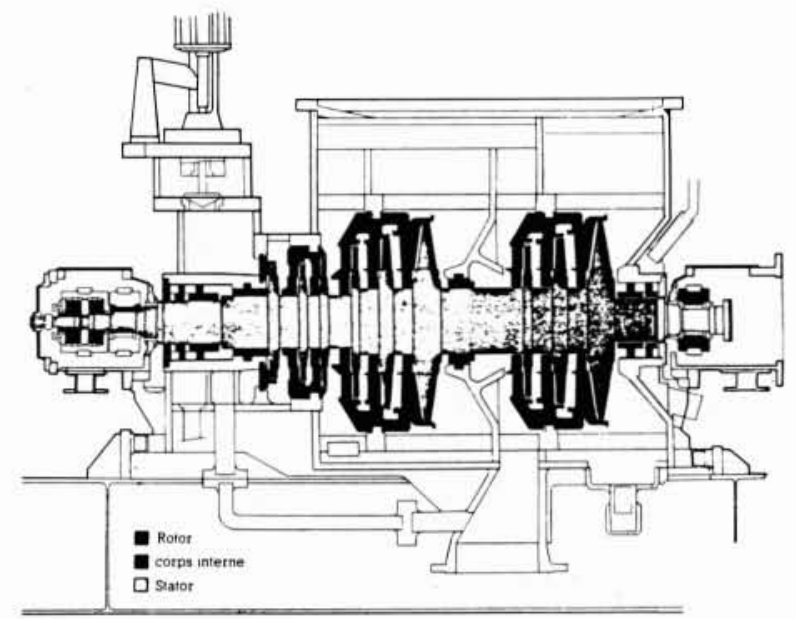

Figure 4. - Coupe de principe de la turbine Alsthom-Atlantique
- les ouvrages de prise d'eau de mer comprenant essentiellement un canal d'amenée, un filtre rotatif et deux pompes verticales;

- le bâtiment usine et la plateforme adjacente où se trouve situé l'ensemble du système de production de vapeur.

Le plan d'implantation (fig. 3) montre la disposition des principaux matériels situés autour du forage producteur BO 2.

Production de vapeur: Elle est constituée principalement du forage $\mathrm{BO} 2$, de deux séparateurs-sécheurs à chicanes, d'un ensemble de tuyauteries, et de silencieux centrifuges pour la détente du fluide diphasique, de l'eau ou de la vapeur sous pression.

L'ensemble des vannes installées sur les circuits sont des vannes à obturateurs sphériques excentrés (papillon excentré) et conçues pour être les moins sensibles possible aux dépôts de silice.

L'ensemble des tuyauteries et des réservoirs sont prévus en acier au carbone avec une surépaisseur de corrosion d'au moins $6 \mathrm{~mm}$. Pour les autres matériels, l'acier à $13 \%$ de chrome (doigt de gant de mesure de température), l'inox $316 \mathrm{~L}$ (instrumentation, petite robinetterie, chicanes), l'uranus 50 (anti-coup d'eau) et le 17.4. PH (axe vannes réglantes) sont utilisés.

En ce qui concerne les silencieux, où la corrosion est particulièrement active, des résines de vinyl-ester armées de fibres de verre (Derakane 470-36) constituent les cheminées d'échappement (compte tenu de la proximité des habitations, une solution de supression des panaches de vapeur est envisagée par l'adjonction de rampes de pulvérisation dans les silencieux actuels).

Groupe turbo-alternateur à condensation: La turbine est située dans un bâtiment usine, au niveau du sol sur un massif en béton pour en faciliter l'accès pour l'entretien.

Compte tenu de la position relative de la turbine et du condenseur, l'échappement de la turbine est dirigé vers le haut.
La vapeur est admise par l'intermédiaire de deux secteurs d'admission Haute Pression et d'une injection Basse Pression, c'est une turbine industrielle à action de type axial, à 9 étages ( 3 H.P. et 2 fois 3 B.P.) tournant à $7904 \mathrm{tr} /$ minute et entraînant l'alternateur par l'intermédiaire d'un réducteur (voir la coupe longitudinale : $f i$ gure 4).

Elle est équipée d'un régulateur de vitesse, d'un régulateur de puissance et d'un limiteur de charge.

Les risques de dépôts et de corrosion sur les surfaces d'échange, et le fait que les condensats de vapeur ne sont pas recyclés, ont fait retenir une solution par condenseur à mélange.

La proximité de la mer et la nécessité de ne pas dépasser, dans tous les cas, une température de rejet de plus de $45^{\circ} \mathrm{C}$ a conduit à prendre l'eau de mer comme fluide de refroidissement.

Les risques de mauvais fonctionnement des pompes d'extraction des condensats en géothermie, et les risques de coups d'eau à la turbine qui en résulteraient, on fait choisir un condenseur à colonne barométrique.

L'ensemble des solutions retenues devraient permettre de garantir un vide d'environ $75 \mathrm{mb}$ à l'échappement de la turbine et un entretien très réduit. De plus, la dilution par l'eau de mer limite les phénomènes de corrosion géothermale dans le condenseur.

Afin de résister aux problèmes de corrosion qui sont le risque majeur en géothermie, différents alliages ont été retenus pour la construction du groupe turbo-alternateur après une série d'essais sur le site (tableau II).

Bâtiment : Le bâtiment est en parpaings pleins de $20 \mathrm{~cm}$ d'épaisseur et il est équipé de tous les dispositifs acoustiques nécessaires pour limiter à $80 \mathrm{~dB}$ (A) la puissance acoustique rayonnée vers l'extérieur.

Station de pompage : Le principe retenu pour la conception de la station de pompage est un canal d'amenée ouvert et l'installation de deux pompes normal/secours qui fonctionnent alternativement. 
Le type d'ouvrage retenu pour la ravine de rejet est un canal ouvert protégé.

Contrôle-commande: Le contrôle-commande est conçu à partir d'une technologie numérique : automates programmables et microprocesseurs.

\section{Aspects économiques}

Le coût d'une installation géothermique dépend de nombreux paramètres, les principaux étant le développement du champ et les caractéristiques géologiques rencontrées : réservoirs d'eau ou de vapeur, débit, température, qualité chimique du fluide géothermal et quantité de gaz dissous.

Il dépend également de la taille des installations de surface et de la technologie employée (simple ou double flash, cycle à fluide volatil), ainsi que de l'éloignement des centrales par rapport aux points de consommation d'électricité.

En ce qui concerne la centrale géothermique de Bouillante, il est à noter que les investissements sont répercutés sur une puissance installée faible et qu'il s'agit d'une centrale prototype qui bénéficie de tous les aménagements d'une centrale classique d'E.D.F. et d'un niveau d'automatisme élevé. De plus, la productivité du réservoir de Bouillante est faible, aussi le coût du $\mathrm{kW}$ installé qui en résulte est-il plus élevé que celui des centrales géothermiques de puissance situées à l'étranger.

A partir des éléments dont nous disposons aujourd'hui, il est possible de chiffrer le coût des installations et d'estimer le prix de revient du KWh produit. Tous les prix indiqués ci-dessous sont exprimés hors taxes aux conditions économiques d'avril 1983.

\section{Installations de surface (à partir de la tête de puits)}

Pour la partie relative aux installations de surface, le total des dépenses se monte à $110 \mathrm{MF}$ qui se décomposent ainsi :

\section{Études : $3 \%$}

\section{Génie civil : $25 \%$}

Tous les bâtiments (y compris G.C. du poste électrique, voirie, clôtures et divers), ouvrages en mer et transport de l'eau de refroidissement, massifs de supportage... etc.

\section{Mécanique : $32 \%$}

Groupe turbo-alternateur, condenseur, tuyauteries, robinetterie, séparateurs, silencieux, pompes, pont roulant... etc.

\section{Électricité : $27 \%$}

Contrôle commande, instrumentation, air comprimé, climatisation, tous travaux électriques, poste d'évacuation d'énergie, Diesel de secours, télétransmission... etc.

Divers : $13 \%$

Frais administratifs, frais de maitre d'œuvre, frais de pré-exploitation, intérêts intercalaires... etc.
Tableau II - Alliages retenus pour la construction du groupe turbo-alternateur

\begin{tabular}{|c|c|c|c|c|c|c|c|c|c|c|}
\hline & & RME & & & & OMPOSIIIOS & $\mathrm{N} \mathrm{CHIMIO}$ & & & \\
\hline DESIGNATION & & & & & & 1 & & & & \\
\hline & AF NOR & Not a & c & $M_{n}$ i & $5 i$ & p & s & $|p \cdot s|$ & $\mathrm{Cr}$ & $\mathrm{Ni}$ \\
\hline Stat or & ${ }^{A 42 \mathrm{CP}}$ & $\begin{array}{l}10 \mathrm{~mm} \\
\text { de } \\
\text { surep. } \\
\text { surp. }\end{array}$ & $\begin{array}{l}0,20 \\
\operatorname{maxi}\end{array}$ & 20,60 & $\begin{array}{l}0,35 \\
\max i \\
\end{array}$ & 0,06 & 0.035 & & & \\
\hline $\begin{array}{c}\text { Rotor } \\
\text { monobloc }\end{array}$ & $\begin{array}{c}26 \mathrm{CNU} \\
17.4\end{array}$ & (Cu:32) & $\leq 0.07$ & $\leq 1$ & $\leq 1$ & $<<0.025$ & $<0,025$ & & $\begin{array}{l}15,5 \\
17,5 \\
17\end{array}$ & $\begin{array}{l}3 \\
5\end{array}$ \\
\hline $\begin{array}{c}\text { Aubes } \\
\text { mobiles }\end{array}$ & $\begin{array}{c}26 \mathrm{CNU} \\
17.4\end{array}$ & $(\mathrm{Cu}: 3 z)$ & \begin{tabular}{|l|} 
\\
\end{tabular} & $\leq 1$ & $\leq 1$ & $<0,025$ & $1<0,025$ & & $\begin{array}{l}15,5 \\
17.5 \\
17\end{array}$ & $\begin{array}{l}3 \\
5\end{array}$ \\
\hline $\begin{array}{l}\text { Diaphrag. } \\
\text { Distribut. }\end{array}$ & $\begin{array}{l}26 \mathrm{CDA} \\
12\end{array}$ & 1 & $\leq 0,08$ & $\leq 1$ & $\leq 1$ & $\leq 0,04$ & $i \leq 0,03$ & & $\begin{array}{l}11,5 \\
13,5 \\
13\end{array}$ & 10,5 \\
\hline $\begin{array}{l}\text { Diaphrag. } \\
\text { bardage }\end{array}$ & $\begin{array}{l}26 \mathrm{CDA} \\
12\end{array}$ & 1 & $\leq 0,08$ & $\leq 1$ & $\leq 1$ & $\leq 0,04$ & $\leq 0,03$ & & $\begin{array}{l}11.5 \\
13.5 \\
13.5\end{array}$ & 0,5 \\
\hline $\begin{array}{l}\text { Diaphrag. } \\
\text { couronnzs }\end{array}$ & $212 \mathrm{cl3}$ & & \begin{tabular}{|l|}
0,10 \\
0,15
\end{tabular} & $\leq 1$ & $\underline{\underline{s}}$ & $\leq 0,025$ & $\leq 0,025$ & & $\begin{array}{l}12 \\
14\end{array}$ & $\leq 0,5$ \\
\hline $\begin{array}{l}\text { Cour onnes } \\
\text { d'et anch. }\end{array}$ & $212 \mathrm{Cl3}$ & & $\begin{array}{l}0,10 \\
0,15\end{array}$ & $\frac{s}{\leq 1}$ & $\begin{array}{l}\underline{s} 1 \\
\underline{s}\end{array}$ & $i \leq 0,025$ & $1 \leq 0,025$ & & $\begin{array}{l}12 \\
14 \\
14\end{array}$ & $\leq 0,5$ \\
\hline $\begin{array}{l}\text { Léchet tes } \\
\text { d'ét anch. }\end{array}$ & $\mathrm{NC} 15 \mathrm{Fe}$ & & $\begin{array}{l}0,15 \mid \\
0,20 \mid\end{array}$ & 1 & 0,5 & & 0,03 & & $\begin{array}{l}14 \\
17\end{array}$ & $\begin{array}{l}72 \\
80\end{array}$ \\
\hline $\begin{array}{l}\text { Bourte de } \\
\text { Sertissage }\end{array}$ & NC15 Fe & & \begin{tabular}{|l|}
0,15 \\
0,20
\end{tabular} & 1 & 0,5 & & 0,03 & & $\begin{array}{l}14 \\
17\end{array}$ & $\begin{array}{l}72 \\
80\end{array}$ \\
\hline $\begin{array}{l}\text { Garnitures } \\
\text { vapeur }\end{array}$ & $212 \mathrm{cl3}$ & & $\begin{array}{l}0,100 \\
0,15\end{array}$ & $\leq 1$ & $\leq 1$ & $\leq 0,025$ & $\leq 0,025$ & & $\begin{array}{l}12 \\
14\end{array}$ & $\{0,5$ \\
\hline $\begin{array}{l}\text { Sieges et } \\
\text { Tiges SR }\end{array}$ & $20 \mathrm{CD} 12$ & $\begin{array}{l}\mid \text { Stel- } \\
\text { lites }\end{array}$ & $\begin{array}{l}0,15 \mid \\
0.25\end{array}$ & $\begin{array}{l}0,4 \\
0,8\end{array}$ & 0,4 & $\leq 0,04$ & $\leq 0,035$ & $i^{0,065}$ & $\begin{array}{l}2,5 \\
3,5\end{array}$ & \\
\hline $\begin{array}{l}\text { Filtre } \\
\text { vapeur }\end{array}$ & $212 \mathrm{cl3}$ & & $\begin{array}{l}0,10 \\
0,15\end{array}$ & $\leq 1$ & $\leq 1$ & $i \leq 0,025$ & $\leq 0.025$ & & $\begin{array}{l}12 \\
14 \\
14\end{array}$ & $i \leq 0,5$ \\
\hline $\begin{array}{l}\text { Corps du } \\
\text { Condenseur }\end{array}$ & A42 CP & $\begin{array}{l}\text { Ebonite } \\
4=0\end{array}$ & \begin{tabular}{l|}
0,20 \\
$\operatorname{maxi}$
\end{tabular} & $\geq 0,60$ & $\begin{array}{l}0,35 \\
\operatorname{maxi}\end{array}$ & 0.04 & 0,035 & & & i \\
\hline $\begin{array}{l}\text { 1nternes du } \\
\text { Condenseur }\end{array}$ & $\begin{array}{l}\text { Cupro } \\
\text { Alumin. } \\
\text { A } 20\end{array}$ & 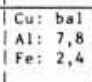 & & $\leq 1$ & 0,005 & $\{\leq 0,015$ & & i & 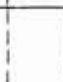 & 0,075 \\
\hline Ejecteurs & $\begin{array}{l}22 \text { CNDU } \\
17.6\end{array}$ & & $i \leq 0,03$ & $\leq 0,03$ & $\leq 0,04$ & & & $i$ & 17 & $\begin{array}{ll}1 & 6 \\
1\end{array}$ \\
\hline
\end{tabular}

On notera que la petite taille de l'installation apparaît en comparant l'importance relative de la part "électricité » par rapport aux matériels mécaniques.

En définitive, l'investissement des installations de surface est de l'ordre de $26000 \mathrm{~F} / \mathbf{k W}$ installé.

\section{Sous-sol}

Pour la partie sous-sol bien que les travaux aient été réalisés entre 1969 et 1977 , une estimation peut être faite pour les 4 kilomètres de forages profonds qui ont été réalisés ainsi que pour les frais de recherches géologiques et les essais de production de vapeur (boucle d'essais).

Les dépenses peuvent être estimées comme suit :

- recherche géologique

$15 \mathrm{MF}$

-3 forages inférieurs à $1000 \mathrm{~m}(350$ à $800 \mathrm{~m}) \quad 15 \mathrm{MF}$

-1 forage à $2500 \mathrm{~m}$

$15 \mathrm{MF}$

- essais de productivité

Total

$50 \mathrm{MF}$

Le total des investissements atteint donc $110 \mathrm{MF}+50$ MF $=160$ MF soit une dépense totale d'environ 38000 $\mathrm{F} / \mathbf{k W}$ installé. 


\section{Prix de revient du $\mathrm{kWh}$ à la production}

A partir de cet investissement le prix de revient du $\mathrm{kWh}$ peut être évalué, en prenant comme hypothèse que les charges d'amortissement sont calculées au taux de $9 \%$ sur 20 ans.

Charges annuelles d'amortissement : Charges d'exploitation :

$4000 \mathrm{~F} / \mathrm{kW} / \mathrm{an}$ $700 \mathrm{~F} / \mathrm{kW} / \mathrm{an}$

Total

$4700 \mathrm{~F} / \mathrm{kW} / \mathrm{an}$

Coût du $k W h$ :

Pour une production de $7000 \mathrm{~h} / \mathrm{an}: \mathbf{6 7} \mathbf{~ c F / k W h}$ pour une production de $5000 \mathrm{~h} / \mathrm{an}$ : $94 \mathrm{cF} / \mathbf{k W h}$

\section{Conclusions}

Dans le cas de la centrale géothermique de Bouillante, et pour un fonctionnement en base sans incident, le coût du $\mathrm{kWh}$ produit est légèrement inférieur à celui des groupes Diesels de $20 \mathrm{MW}$ installés à Jarry.

\section{Bibliographie}

(1) Exploration géothermique en Guadeloupe, J. Demians d'Archimbaud et J.P. Munier-Jolain - Annales des Mines, mai 1971.

(2) Documents E.D.F. sur la centrale géothermique de Bouillante Direction des Études et Recherches, P. Jaud

Direction régionale chargée des Départements d'Outre Mer, D. Lamethe

Région d'Équipement Alpes-Marseille, D. Boyer

\section{Discussion}

Président : M. J. VARET

M. LEDOUX demande si la présence de silice dans l'eau pose un problème pour la circulation dans la turbine.

M. JAUD. - Plus on détend le fluide, plus on le refroidit et plus les dépôts sont importants. Au niveau des vannes sur la phase liquide, on peut trouver des dépôts de silice de plusieurs centimètres ce qui nécessite de les nettoyer tous les ans. En ce qui concerne la turbine qui est traversée par la vapeur on pense qu'il sera nécessaire de l'ouvrir pour nettoyage tous les ans ou tous les deux ans.

M. MARTIN. - Avez-vous noté la présence de métaux lourds dans le fluide?

M. JAUD. - S'il y en a, c'est en faible proportion.

M. MARTIN. - De tels rejets posent-ils des problèmes de pollution?

Mme LAMETHE. - Autour de l'endroit où l'on rejette il y a énormément de sources naturelles qui sont de même type et qui ont pratiquement la même composition que les rejets.

LE PRÉSIDENT remarque que l'économie de ce type de projet dépend beaucoup du temps que l'on met à le réaliser, puisqu'on immobilise un fort capital en forages. Les délais qui ont eu lieu à Bouillante entre les forages et la réalisation sont trop prohibitifs pour qu'un projet mené dans ces conditions soit économique, en tout cas pour celui qui investit dans le forage.

Mme LAMETHE ajoute qu'à Bouillante une part importante du surcoût d'installation est due à des problèmes d'environnement.

LE PRÉSIDENT. - Une autre implantation aurait probablement permis d'installer une turbine à contre pression qui permettait d'utiliser la vapeur plus rapidement.

Mme LAMETHE. - La proximité des habitations ne l'a pas permis.

Un Participant. - Est-ce que les turbines sont de fabrication française et vous donnent-elles satisfaction?

M. JAUD. - Elles sont construites par Alsthom-Rateau. On n'a pas suffisamment d'expérience pour connaître tous les problèmes liés à la géothermie mais il y a, dans le monde, des expériences qui montrent que les turbines peuvent tourner plus d'une dizaine d'années à condition d'effectuer un entretien périodique.

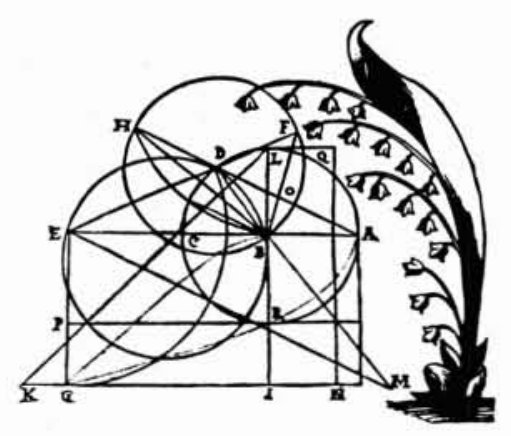

